\title{
Aniversario XXV de la Revista Salus.
}

https://doi.org/10.54139/salus.v25i3.124

Estimados amigos, hemos querido cerrar el volumen 25.3 de Salus, periodo septiembre-diciembre, del presente año, dándole la bienvenida al año aniversario XXV de nuestra Revista Salus.

En esta oportunidad debemos resaltar que en el presente volumen hacemos gala de un verdadero resumen de los más importantes avances en investigación de COVID19.

Son pocas las pandemias conocidas que hayan marcado tanto nuestra historia en medicina y la OMS el 11 de marzo de 2020, la declara pandemia a la "enfermedad por coronavirus", pasando de ser una emergencia de salud en el mundo con una alta tasa de propagación, a convertirse en un problema de salud pública.

A través de la Dra. Vizzi, del Instituto Venezolano de Investigaciones Científicas -IVICcomo organismo administrador de proyectos de desarrollo tecnológico en las áreas médico-salud, industrial, química, ambiente y academia, se organizaron las revisiones más actualizadas hasta el momento de esta enfermedad.

Queremos expresar nuestras más sinceras felicitaciones al excelente grupo de investigadores de diversas instituciones nacionales e internacionales, que hicieron posible esta colección. Agradecemos al: Instituto de Virología Humana. Escuela de Medicina de la Universidad de Maryland. Baltimore MD. Estados Unidos. Instituto de Inmunología. Facultad de Medicina. Universidad Central de Venezuela. Sociedad Venezolana de Infectología e Instituto Médico La Floresta. Caracas

Laboratorio de Virología Molecular Laboratorio de Biología de Virus

Unidad de Microscopía electrónica del Centro de Microbiología y Biología Celular Laboratorio de Fisiopatología. Sección Inmunogenética. Centro de Medicina Experimental Miguel Layrisse. IVIC. Caracas. y a la catedra de Semiología del Departamento Clínico Integral del Norte. Escuela de Medicina.

Facultad de Ciencias de la Salud. Universidad de Carabobo. Valencia, Venezuela

Les comunico que ya estamos en la plataforma OJS/PKP. Seguimos en la búsqueda del prestigio nacional e internacional de Salus y convertirla en orgullo de docentes, estudiantes y personal administrativo y obrero, de la Facultad de Ciencias de la Salud, de toda la Universidad de Carabobo; aspiramos que por su calidad científica y humanística, sea referencia obligada en estas disciplinas.

Marisol Garcia de Yeguez Editor Jefe 